\title{
Cabellos y barbas: narrativas de hombres de clase media alta limeña
}

\section{Liuba Kogan}

(iD) https://orcid.org/0000-0001-9711-8627

Universidad del Pacífico, Perú

Kogan_1@up.edu.pe

RESUMEN

En este texto analizamos las narrativas sobre el arreglo del cabello y la barba de catorce hombres de 27 a 40 años de edad de clase media alta limeña, así como de barberos y administradores de barberías. Realizamos entrevistas en profundidad y un análisis de su contenido temático e interpretativo. Encontramos que los entrevistados emplean diversas estrategias para evitar ser feminizados debido a su interés por arreglarse. A su vez, estos hombres buscan alinear su corte del cabello o la barba con la ropa que usan y su tipo de cuerpo, con el fin de estar bien presentados, lo que en el Perú se vincula fuertemente con narrativas raciales, de género y de clase. Palabras clave: cabello, barba, género, raza, clase media alta.

\section{Hair and beards: narratives of upper-middle-class men from Lima}

\section{ABSTRACT}

In this paper we explore the hair and beard grooming narratives of fourteen upper-middle-class men aged between 27 and 40 years from Lima, as well as those of barbers and barbershop managers. We conduct in-depth interviews and analyze their thematic and interpretive content. We find that the interviewees use various strategies to avoid being feminized by their interest in grooming. At the same time, the subjects seek to match their hairstyle and/or beard with their clothing and body type in order to appear well turned-out; in Peru, this practice is strongly linked to racial, gender and class narratives. Keywords: hair, beard, gender, race, upper-middle-class. 


\section{INTRODUCCIÓN ${ }^{1}$}

El cabello es un símbolo poderoso de la identidad individual y grupal dada su naturaleza visible y pública (Synnott, 1987) y se puede moldear a voluntad y con relativa facilidad su largo, color, estilo, cantidad, tipo de afeitado, atado y textura (Cox, 2008). Así, debido a su constante crecimiento, manipulación y eliminación, el cabello resulta difícil de ignorar (Cheang, 2008). De tal modo, el cabello - y, por añadidura, la barba - permiten marcar singularidades personales y grupales en tanto posibilitan vínculos inter e intra grupales en virtud de variables como la raza, la clase social, el género, la sexualidad y la edad, entre otros (Moore y Casper, 2015; Barber, 2008; Clarke y Griffin, 2008).

A pesar de la relevancia de la cara en tanto locus para la construcción de la apariencia personal y a la cabeza como símbolo social (Fuller, 2001), y a la centralidad de ambos para la interacción social (Hall, 2015), las ciencias sociales han prestado relativamente poca atención al estudio del cabello y de la barba de los hombres occidentales. Sin embargo, las investigaciones sobre la apariencia masculina son cada vez mayores (Hall, 2015; Barber, 2016; Weiner, 2019). No en vano se hacen evidentes en la actualidad los productos cosméticos y de higiene promocionados para hombres que se hallan en los pasillos de los supermercados, los que se venden en línea o los que se publicitan en las redes sociales (Villa, 2015; Santos y Pereira, 2019). En el Perú - a pesar de ser un mercado emergente y conservador - encontramos un significativo aumento del consumo de productos de higiene y belleza masculina: su consumo per cápita pasó de US\$ 12,9 en el año 2005 a US\$ 38,5 en el año 2019 (Euromonitor International, 2020), y entre el año 2017 e inicios de 2020, el número de barberías creció en 20\% (La Cámara, 2020). Agreguemos que, según los administradores y barberos que entrevistamos, la inmigración venezolana reciente hacia el Perú ha incidido

1 La investigación que llevé a cabo fue financiada por la Universidad del Pacífico. Mi gratitud a Franco Salazar por su asistencia en la investigación y su aporte en la discusión de las ideas que se encuentran plasmadas en el texto. Mi agradecimiento a los entrevistados, quienes generosamente compartieron sus saberes y experiencias. 
en la oferta de servicios para el arreglo del cabello. Esto se debe a la cultura de cuidado masculino común entre los hombres venezolanos y a que los barberos de dicho país cuentan con una amplia experiencia en dicho rubro.

Con relación a los tipos de establecimientos en los que se atienden los hombres para cortar cabellos y barbas, señalemos que en la primera década del siglo XXI se consolidaron en Lima tres tipos inéditos: las barberías urbanas, las barberías clásicas y los salones masculinos. Las barberías urbanas se inspiran en las subculturas musicales del reggaetón, el trap y el hip hop, y sus cortes imitan a los de sus artistas más conocidos. Estas barberías fueron las primeras que se establecieron en la capital, especialmente en zonas populares, según nos relató Luis Ángel Pérez - conocido como El Turco- administrador de una de las cadenas de barberías clásicas más antiguas del país. Posteriormente, señaló que la oferta de barberías se extendió a sectores más acomodados en la modalidad de barberías clásicas. Estos establecimientos se caracterizan por contar con una decoración retro o vintage que remite a las barberías de las décadas de 1950 a 1970 y se especializan en cortes clásicos de la barba y el cabello realizados con máquinas (Escalante, 2019). En los últimos años han aparecido salones masculinos que se identifican por su decoración industrial, minimalista o pop art, en los cuales se realizan cortes contemporáneos con tijeras y que se enfocan en el estilismo. Su público objetivo, al igual que el de las barberías clásicas, son hombres con una posición socioeconómica acomodada, como comenta Mateo a partir de su experiencia de años como administrador de uno esos establecimientos limeños.

Cabe señalar que en el Perú las investigaciones empíricas sobre el arreglo corporal y la belleza masculina no son abundantes, como tampoco las referidas al cabello y la barba. Destacan el libro de Fuller (2001) sobre masculinidades en tres ciudades del Perú y su artículo sobre el cuerpo masculino y la belleza (Fuller, 2017). En este resalta la propuesta de estudiar el cuerpo del hombre no solo como materia (órganos sexuales y musculatura), sino como apariencia; esto es, analizar la cara y los adornos corporales. Por su parte, Kogan y Galarza (2014) exploran la percepción de la presencia corporal y la discriminación de jóvenes universitarios en cuatro ciudades del Perú. Es pionera, por otro lado, la investigación de Villa (2015) sobre las prácticas de arreglo corporal juvenil y el desarreglo meticuloso en sectores altos de Lima. Sobresale la reciente tesis de Centurión (2021) sobre barberos en Lima, la belleza masculina y la hegemonía visual en torno al pelo y el vello facial, en la que se pregunta de qué manera los discursos de los barberos y la visualidad de las barberías prometen al hombre el cumplimiento de ideales sociales relacionados con la raza y la clase. Consideremos 
a su turno el estudio de Neila Boyer (2006) que da pistas sobre la importancia del cabello en la cosmovisión andina y las pesquisas de Junyi (2019) y Gonzales Saavedra (2013) acerca de los rituales y el cuerpo indígena amazónico. Sobre el cabello femenino en el Perú encontramos la investigación de Janampa (2013) en torno a las tensiones emocionales que viven mujeres de clase media alta cuando se tiñen el cabello de rubio, y el trabajo de Álvarez (2018) en el que se analizan propuestas artísticas alrededor del cabello femenino. En ese contexto, el trabajo que proponemos se suma a la escasa producción académica sobre la temática de la apariencia masculina y el cabello y la barba.

Lo que motivó el trabajo que presentamos fue el visible aumento de barberías clásicas en la ciudad de Lima y el aparente interés de los hombres de clase media alta por cortar barbas y cabellos en ellas. Esto nos llevó a analizar las narrativas de hombres de clase media alta sobre el trabajo del cabello y de la barba, de cara a las relaciones de género, raza y clase social, en tanto el cuerpo expresa y es expresión de relaciones de poder y de creatividad. Para estudiar las narrativas de hombres de clase media alta limeña dejamos de lado a aquellos clientes de barberías urbanas que no los tienen como público objetivo. Y, a su vez, consideramos barberías clásicas en detrimento de los salones masculinos, ya que en aquellas se trabajan tanto barbas como cabellos. Queda como tarea pendiente la investigación de los clientes de las barberías urbanas y de los salones masculinos.

Partiendo del enfoque de la Teoría Fundamentada (Glasser y Strauss, 1967), analizamos las narrativas de catorce clientes — de 27 a 40 años de clase media alta limeña que asisten a barberías clásicas entre dos a tres veces al mes- que obtuvimos a partir de entrevistas en profundidad. Elegimos este grupo de edad en tanto se trata de hombres con profesiones en desarrollo ascendente, que tienen interés en su apariencia e ingresos para costear el tratamiento del cabello y la barba con regularidad. Cabe señalar que adicionalmente sostuvimos entrevistas con cuatro barberos y tres administradores de barberías en su calidad de intermediarios culturales; esto es, quienes interpretan lo que está de moda y lo que, en diálogo con los clientes, les ofrecen².

El trabajo de campo lo realizó un asistente de investigación, hombre cisgénero, quien logró contactar a tres administradores de barberías quienes accedieron a la entrevista. En dos casos la aceptación se dio gracias al afán de colaboración con el joven investigador en el entendido de que se trataba de una investigación

2 A lo largo del texto nos referiremos a los catorce entrevistados, que son clientes de las barberías, indistintamente como clientes o entrevistados. 
académica, mas no una de mercadeo. La última entrevista se logró gracias a un conocido del entrevistador, quien coordinó el encuentro entre este y el administrador. No fue fácil contar con los administradores como participantes de la investigación, porque no siempre se encontraban en los establecimientos que administran o porque desconfiaban del investigador en tanto temían que la información solicitada fuera usada por la competencia.

A dos barberos se les contactó en dos barberías en las que se entrevistó a sus administradores, previo permiso. Con ellos se conversó en un café fuera de sus horas de trabajo. En un caso el entrevistador solicitó el servicio de barbería en un establecimiento y logró que el barbero le diera una entrevista al finalizar su jornada de trabajo. Al último de los barberos se accedió a través de un conocido.

A los clientes de barberías se accedió por la técnica de bola de nieve bajo la premisa de que fueran de clase media alta $^{3}$, que asistieran por lo menos dos veces al mes a una barbería y cuyo corte de cabello costara como mínimo 25 soles al mes. Con las catorce entrevistas se saturó la muestra. Se transcribieron los audios de las veintiún entrevistas (clientes, administradores y barberos), se codificó la información y se procedió a su análisis temático e interpretativo.

Todos los participantes en la investigación dieron su consentimiento informado. Los nombres de los informantes se mantienen en el anonimato, por lo cual en el texto se utilizan nombres ficticios. La investigación se llevó a cabo bajo el Código de Ética del Centro de Investigación de la Universidad del Pacífico.

\section{Lista 1. Entrevistados}

\begin{tabular}{lcl}
\hline Nombre & Edad & Actividad a la que se dedican \\
\hline Alberto & 34 & Administrador de empresas \\
Sebastián & 29 & Artista del tatuaje \\
Ignacio & 39 & Publicista y músico \\
Enrique & 32 & Marketero \\
Ricardo & 31 & Administrador de empresas \\
Daniel & 27 & Nutricionista e influencer \\
Óscar & 30 & Abogado \\
Javier & 40 & Ingeniero electrónico \\
Ronaldo & 36 & Agente de viajes \\
Hernán & 39 & Administrador de empresas \\
Carlos & 35 & Abogado \\
\hline
\end{tabular}

Se tomó como referencia el distrito de residencia y el lugar de trabajo. Se consideró, a su vez, la percepción de los participantes que recomendaban a otros, a los que se les pedía que fueran «de la misma clase social». 


\begin{tabular}{lcl}
\hline Nombre & Edad & Actividad a la que se dedican \\
\hline Franco & 29 & Economista \\
Rubén & 30 & Abogado \\
Renato & 32 & Comunicador \\
Bruno & 31 & Barbero \\
Daniel & 28 & Barbero \\
Francisco & 29 & Barbero \\
Andrés & 32 & Barbero \\
Mateo & 42 & Administrador de barbería \\
Antonio & 41 & Administrador de barbería \\
Esteban & 37 & Administrador de barbería \\
\hline
\end{tabular}

\section{EL CABELLO Y LA BARBA COMO CARTAS DE PRESENTACIÓN}

«El pelo es como el CV», sentencia Ronaldo (agente de viajes), porque da cuenta de las singularidades de cada individuo. Pero el cabello y la barba no solo son una carta de presentación en tanto dejan vislumbrar hábitos, acciones o elecciones cotidianas, sino que como materia corporal inherente a la persona actúan como símbolos de la historia familiar. Francisco (barbero) lo explica:

La ropa, los tatuajes, los lentes, los aretes, los piercings, las cadenas, todo es ajeno, ¿me entiendes? Tu cabello, lo tienes ahí por algo, tu barba si la tienes, la tienes ahí por algo, y eres tú, es tu familia, son tus antepasados, es tu registro histórico también, y es parte de tu vida.

En ese sentido, podríamos decir que los hombres pueden estar en el mundo con una narrativa de sí mismos encarnada en el corte del pelo. Además, en algunos casos la materialidad del cabello y de la barba pueden remitir al pasado, que actúa como referente del sí mismo. Pero no solo eso, los entrevistados señalan que es posible transformar(se) a partir del trabajo del cabello o de la barba, pues la apariencia del rostro cambia significativamente con los diversos cortes, peinados y tratamientos. No obstante, algún nivel de calvicie puede afectar la autoestima, la vida social y la estabilidad emocional de los hombres (Alfonso, Richter-Appelt, Tosti, Miguel y García, 2005). Siguiendo esa línea argumentativa, el trabajo sobre el cabello y la barba serían deseables porque permiten ocultar defectos o resaltar virtudes, «[...] te puede hacer más viejo, te puede hacer más flaco, te puede hacer ver más joven, te puede hacer ver más gordo, te puede hacer ver más flaco, más alto, más chato» (Ignacio, publicista). Entonces, la posibilidad de transformar la imagen de la cara como la del cuerpo, a través del trabajo del cabello o de la barba, tiene un 
impacto fundamental en la experiencia vital de los entrevistados. Andrés (barbero) sentencia «[un buen corte te] puede cambiar el día y hasta la vida, sí o sí̀».

El concepto de buen corte es un leit motiv en las narraciones de barberos, clientes y administradores, pues marca el objetivo de la asistencia a las barberías, esto es, en última instancia, la construcción de una narrativa positiva de sí mismo. El buen corte implica, pues, tres elementos principales: el orden, el cambio de la apariencia y la comodidad.

En primer lugar, se considera que el cabello y la barba crecen desordenados, pues esa es su naturaleza, y que la función del hombre es encauzarlos con el fin de adquirir una buena apariencia. El desorden del cabello y de la barba son indicadores de abandono y de falta de armonía, e incluso — en un extremo- de la posibilidad de adquirir la apariencia de un vago o de un loco. En segundo lugar, los barberos buscan que el corte sea fresh, es decir, que el hombre pueda sentirse fresco o diferente a partir de un cambio en su rostro. Daniel (barbero) comenta que los clientes «[se dan cuenta de que] necesitan un buen corte, cambiar su apariencia, probar nuevas cosas y que esto los ayude a verse mejor y tal vez incluso a sentirse mejor». En tercer lugar, un buen corte es aquel que se puede mantener en casa de modo similar al logrado en las barberías sin tener que luchar con mechones que tapan la cara ni batallar contra una barba mal cuidada, para lo cual los barberos suelen recomendar productos de diferentes marcas. Si no se logra mantener el resultado original, asoman la fealdad, pero sobre todo la incomodidad. Así, Daniel (barbero) menciona enfáticamente que, si no se tiene la barba bien cuidada, «te empieza el picor, te estás rascando, o por ahí se ha metido un pelo del bigote en la nariz y cosas así que son bien feas».

En síntesis, el buen corte transforma al sujeto para bien, porque permite construir una apariencia (re)actualizada de sí mismo, al encauzar el desorden del pelo - que es su naturaleza - y lo mantiene con comodidad hasta que vuelva a necesitar una nueva intervención.

\section{EL ENCUENTRO CON LA VANIDAD Y LAS ESTRATEGIAS DE DESFEMINIZACIÓN}

Los entrevistados señalan que su generación es más vanidosa que la de sus padres y abuelos, porque los hombres contemporáneos pueden preocuparse por su imagen corporal y acicalarse haciendo uso de los productos que ofrece el mercado para ese fin sin ser tildados necesariamente de homosexuales o afeminados. Andrés (barbero) comenta en ese sentido que «mientras más me cuido más mujeres 
consigo y más masculino me siento». La generación de los padres y abuelos indica - relacionaba la masculinidad con la rudeza y con los cortes clásicos, y de ningún modo podía aventurarse a utilizar productos para mejorar su apariencia física y facial. Sin embargo, en la actualidad, según los clientes, barberos y administradores, el trabajo de la apariencia ha pasado a ser una necesidad.

Anotemos que las preocupaciones de los hombres sobre la apariencia y la desfeminización respecto a sus cuerpos tienen origen en el contexto del capitalismo tardío, en tanto a los trabajadores de cuello blanco en el área de servicios se les exige una apariencia ordenada y limpia, y a estar bien vestidos para relacionarse con clientes y colegas (McDowell, 2005). Así que, para la mayoría de los hombres «[...] ya no es suficiente $[\ldots]$ trabajar duro, $[\ldots]$ también deben verse bien» (Barber, 2008, p. 459).

Así, en la actualidad, el cabello y la barba representan materia de poiesis, esto es, de experimentación y creatividad para lograr una estética que los haga sentir bien consigo mismos. Cabe indicar que las imágenes masculinas contemporáneas, como en el caso de las mujeres, se ven acompañadas del mensaje del cuerpo como de un «proyecto» en el que los hombres tienen la posibilidad de automejora (Grogan, 2010). De esta forma, se alimenta la constante búsqueda de imperfecciones, lo que trae consigo inseguridad respecto al propio cuerpo, que solo puede ser superada temporalmente con el mantenimiento de este. Y así, el trabajo de la apariencia se instala en la vida de los hombres, aunque no sin otra inseguridad: el temor de la feminización.

Cabe recordar que los clientes, barberos y administradores diferencian la belleza de la buena apariencia, en tanto conciben a esta última como lejana de los conceptos de femineidad y de metrosexualidad, entendida como vanidad homoerótica (Hall, 2014). En ese sentido, Esteban, administrador de una barbería, comenta en un intento de desfeminizar el trabajo de la vanidad que «la belleza es difícil de definir, pero se puede encontrar en la ropa o en un jarrón de flores [...] lo que el hombre adquiere en la barbería no es belleza sino buena apariencia».

Los hombres con quienes conversamos acuden a la barbería entre dos y tres veces por mes con la finalidad de mantener en forma el corte del cabello o la barba. Incluso algunos comentan que van a la barbería cuando asisten a una reunión de trabajo o evento social para tener el pelo o la barba recién cortados y peinados $\mathrm{y}$, de esa forma, lograr la mejor apariencia posible. No obstante, se preocupan por diferenciarse del hombre metrosexual (excesivo y vanidoso) pues la buena apariencia significa contención y orden. Los entrevistados también establecen límites respecto de los mariconcitos, hombres feminizados y ridiculizados, evitando intervenir ciertas partes del cuerpo que se encuentran marcadas. 
Por ejemplo, un grupo de entrevistados considera que los hombres masculinos no pueden hacerse la manicura, pero sí depilarse las cejas. Pero más allá de las narrativas de la feminización y de la vanidad excesiva y homoerótica del metrosexual, los clientes de las barberías concuerdan en que el hombre tiene derecho a arreglarse. De modo tal que, a lo largo del tiempo, cada vez más hombres utilizan productos cosméticos y de cuidado personal, lo que incluye aquellos especializados para el cabello y la barba. Pero como señalamos, los hombres «a pesar de tener un interés en su apariencia [...] tienen que simultáneamente repudiar cualquier interés en su apariencia» (Hall, 2014, p. 30). Por ello, «trabajan para cultivar una apariencia que, paradójicamente, no llame la atención hacia su interés sobre la apariencia» (Weiner, 2019, p. 6), es decir, que se vea natural (Ocejo, 2017). Así, se considera que la manipulación del cabello y de la barba representa una forma de cuidar la apariencia, el aseo y el logro de verse bien, y no de caer en la pura vanidad. De hecho, los productos para el tratamiento del cabello no se ofrecen de la misma manera que los de las mujeres, pues se apoyan en valores, imágenes y saberes masculinos (Cheong y Kaur, 2015). En cierta publicitaria se promueve la diferencia binaria entre géneros para marcar el rechazo a la homosexualidad y así evitar que los hombres se sientan amenazados en su identidad sexual (Edwards, 2003).

\section{EDUCACIÓN E INVERSIÓN}

Los hombres con los que conversamos consideran a las barberías lugares agradables a los que asistir e, incluso, algunos los ven como espacios para la relajación y el esparcimiento. Todo ello se debe a la calidad de servicios que les brindan, al ambiente que encuentran y al profesionalismo de los barberos. Los clientes llegan a las barberías recomendados por familiares o amigos y en ellas conoce a barberos a los que, en un número de casos, siguen mientras se mudan de una barbería a otra, incluso en distritos que les quedan lejos de la casa o del trabajo. En otros casos, no tienen inconveniente en ponerse en las manos del barbero de turno, pues confían en los profesionales de las barberías a las que acuden.

Un conjunto de entrevistados acude a la barbería con una idea previa sobre el corte de cabello o barba que se quiere realizar. Otros esperan recibir el comentario del barbero para ver si la idea con la que llegan es adecuada. Un tercer grupo se pone en las manos del barbero a la espera de que sea él quien sugiera lo que se debe hacer con su barba o cabello. Aquellos que llegan a la barbería con una propuesta sobre la forma en que quieren cortarse el cabello, por lo general, toman 
los modelos de jugadores de fútbol extranjeros o de artistas de películas, series o músicos. La globalización facilita que circulen imágenes por medio de las redes sociales, lo que multiplica las fotos de cortes que se pueden imitar. Otras veces, los hombres utilizan cartillas con modelos de peinados que ofrecen las barberías, mientras que, quienes tienen una idea, la ponen a consideración del barbero e inician un proceso de negociación. Dicha negociación se materializa en algunos casos a partir de dosis de confianza o como señala Hernán (administrador de empresas) «de la energía que tiene el barbero, el feeling o la onda que tiene $[. .$.$] eso de repente influye mucho en el corte del cabello o la barba».$ Para Bruno (barbero), se trata de llegar a un punto medio respecto de lo que él quiere de acuerdo con su experiencia y lo que el cliente busca. Incluso algunos entrevistados quieren aprender el lenguaje especializado que utiliza el barbero, así como los tipos de cortes para solicitar la siguiente vez el mismo look.

El barbero funge de intermediario cultural respecto del hombre que asiste a una barbería con la finalidad de cortarse o tratar el cabello o la barba. Esto es, lo ayuda a encontrar un estilo o afinar el que tiene acorde a las tendencias de la moda y de sus características físicas y faciales. Para ello, el barbero señala lo que se debe hacer de acuerdo a las peculiaridades del cliente. De esta manera, averigua su ocupación, indaga acerca de su personalidad, analiza su imagen corporal (ropa, accesorios, peso) y pregunta por todo aquello que — según los barberos - les permite tener una idea de quién es:

Pucha, como que trato de indagar un poco en su vida. No profundamente, no pensando mucho, sino en qué trabaja, a qué se dedica, qué música escucha. Porque en realidad, a través de eso como que uno puede tener más o menos un criterio o ir sacando una línea de cómo es que un cliente puede llevar el corte (Bruno, barbero).

Por otra parte, el barbero analiza la estructura ósea de la cabeza, la forma de la cara, las características de su cabello y la barba del cliente con el fin de hacer un buen corte. Antonio, el administrador de una barbería, comenta que capacita permanentemente a sus barberos, ya que señala que no solo se trata de manejar una máquina sino además se debe conocer las características del cabello, de los huesos de la cabeza y «saber cómo tu rostro encaja en un corte o no, qué hacer si tiene la cabeza redonda, si tiene la cabeza cuadrada, si es orejón o no es orejón». Y los entrevistados valoran mucho que el barbero conozca las características de la cabeza, el cabello y la barba, así como su textura, densidad y remolinos, lo que determina la calidad del corte. «Cada persona es única y por ejemplo en la 
barba yo tengo dos remolinos, que, si el barbero no los reconoce bien, me va a cortar mal la barba, por eso me gusta cortarme con él» (Renato, comunicador)

Luego de tener una idea de las características del cliente respecto de su aspecto físico y de las características de su cabello o barba, así como su personalidad, el barbero propone un estilo. Esto es, la manera en que podría verse el hombre que tiene al frente para posteriormente proponer un tipo de corte.

La segunda función del barbero es la educativa, esto es, como señala Esteban (administrador de barbería) «somos educadores de la apariencia masculina». El barbero enseña al cliente la manera de tratar su cabello o barba, en tanto racionaliza y naturaliza el trabajo de la belleza, desfeminizándola. Es decir, le muestra los productos adecuados para arreglar su cabello o la barba, además de la forma de trabajarlas para que los peinados queden iguales a los conseguidos en la barbería (la manera de secarlos, de peinarlos y de aplicar los productos). Por último, le brinda algunos consejos sobre los cuidados de la piel, la depilación de las cejas, etc.

Entonces lo que hacemos es educar, «Hey broder, deja de utilizar cosas de mujeres y utiliza cosas de hombres», porque te van a perdurar el cabello, te van a cuidar la piel, te van a cuidar los folículos de la barba, o sea te estamos cuidando (Esteban, administrador de barbería).

Así como la barbería representa un espacio de educación para conseguir buena apariencia, el buen corte es considerado por los entrevistados una buena inversión, porque se desarrollan estilos de cortes que van de la mano — según administradores y barberos - a una buena imagen personal. Y, porque esos buenos cortes impulsan las carreras profesionales de los clientes. Antonio, administrador de una barbería, lo dice con claridad, «nuestro público son jóvenes que están en pleno ascenso de su carrera profesional y su imagen es bien importante, porque eso les ayuda a catapultar su profesión».

Resulta interesante notar que, para algunos barberos y administradores, conseguir una imagen adecuada o estilo personal es un proceso que se realiza poco a poco en la medida en que el cliente va confiando en su barbero, y este le va haciendo recomendaciones en cada visita a la barbería. Se trata, entonces, como señala Esteban (administrador de barbería), de «construir imágenes, desarrollar personas». Sobre todo, en el caso de las barbas, los hombres deben mantenerlas utilizando diversos productos, dado que pueden lucir desordenadas, oler mal o generar enfermedades en la piel. El mantenimiento de la barba consiste, pues, en el uso de productos para el aseo, así como otros para lograr suavidad, brillo y mejorar su textura. 
Al hablar de la importancia de la apariencia, los entrevistados se refieren constantemente a su importancia dentro del ámbito laboral. Concuerdan en que la imagen no lo es todo para ascender en sus trabajos, pero influye estar bien presentados para las entrevistas laborales, para las relaciones con los clientes y para la evaluación positiva de sus contrapartes. Igualmente, tener buena apariencia ayuda en las interacciones sociales, ya que se siente confianza, seguridad y eleva la autoestima personal.

En algunos casos, la barba puede resultar una herramienta poderosa para aquellos jóvenes que buscan respeto en el ámbito laboral en la medida en que agrega años. Por ejemplo, Alberto (administrador de empresas) señala que la barba le permitió dejar de ser invisibilizado en su quehacer profesional: «cuando me dejé la barba, la gente de repente dejó de verme como un niño y ya me veía como un adulto, y como te comentaba, antes me decían "quiero hablar con tu jefe"». Sin embargo, las barbas pueden generar rechazo entre jefes y compañeros de trabajo cuando tiene apariencia desordenada. Incluso, algunos entrevistados refieren que son los jefes quienes piden a sus subordinados que se corten la barba cuando esta es muy larga como condición para seguir laborando debido a problemas con la imagen corporativa.

Un buen corte de barba no solo se considera una buena inversión en el ámbito laboral, sino también en el de las relaciones con el sexo opuesto, según relatan algunos entrevistados. Ello, en la medida en que creen que sus barbas les proveen sex appeal y que por ello las mujeres los prefieren frente a quienes no las tienen. Ricardo, administrador de empresas, sentencia: «la mujer valora al hombre con barba como si hubiese una fuerza de atracción».

Como mencionamos, los entrevistados señalan que los buenos cortes y su mantenimiento les brindan seguridad personal, se sienten atractivos y confiados y con la autoestima elevada. Además, les permite sentirse contemporáneos, es decir, personas atentas a los estilos que consideran en boga. Aún más, combinan lo que les queda bien dependiendo de quiénes son, a qué se dedican y a la imagen que desean proyectar. En ese sentido, el corte y el mantenimiento del cabello y de la barba permiten pensar en un proyecto personal para la construcción de la imagen corporal. 


\section{BIEN PRESENTADO Y ALINEADO}

Los entrevistados, barberos y administradores consideran que estar bien alineado es una condición necesaria para estar bien presentado. Y que ello funciona como una llave maestra para entablar relaciones sociales exitosas, en tanto permite que las personas alcancen sus metas personales y laborales.

Estar bien alineado significa tener una presencia impecable, esto es, que combinen a la perfección el tipo de ropa, las características del cuerpo y, además, el cabello y la barba. Dicha combinación exige que concuerden la ropa y el cuerpo en sus dimensiones y forma, y que, a la vez, armonicen con el tipo de corte de cabello y de barba. La condición de impecable - esto es, llevar con orden y armonía ropa, cuerpo, cabello y barba - también requiere pautas de aseo y buen olor.

Cabe resaltar que, según algunos entrevistados, es posible que en cualquier clase social un hombre pueda estar bien presentado, porque no es necesario utilizar ropa cara de marca y porque se puede tener un buen corte de pelo si se encuentra un barbero que no cobre caro. Pero, a pesar de la declaración de principio de que se puede estar impecable en cualquier clase social, los entrevistados proponen que, en términos generales, las clases sociales bajas suelen tener estilos estrafalarios y modas identificables — esto es, mal gusto- $\mathrm{y}$, como consecuencia, estar mal presentados. Saber combinar y armonizar - es decir, alinear cuerpo, ropa, cabello y barba - haría de los hombres de las clases medias altas los poseedores de estilos sofisticados y, por tanto, poseedores de buena apariencia.

«Córtame como Pizarro y no como Cuevita», le pide el abogado Rubén a su barbero, reafirmando su sofisticación y buen gusto. Christian Cueva y Claudio Pizarro son futbolistas peruanos. La imagen de Pizarro, considerado blanco, se asocia con su profesionalismo como jugador de uno de los más importantes equipos alemanes y como un inteligente inversionista, mientras a Cueva, de rasgos mestizos y clase baja e identificable por sus cortes de cabello llamativos, se le relaciona con escándalos ligados a la fiesta y a la bebida antes que a sus logros deportivos. Resulta interesante que el ejemplo de 'Cuevita' haya aparecido repetidas veces entre los entrevistados para establecer la relación entre la clase social, la raza y el mal gusto.

Uno de los registros narrativos en que se entiende la dicotomía entre buena apariencia y mala apariencia es la manera en que los barberos y los administradores racializan los tipos de cabello al clasificarlos como «peruanos» y «europeos». Los primeros se consideran poco maleables y se caracterizan de modo negativo 
como chutos, greñudos o trinchudos ${ }^{4}$, y los segundos se describen de modo positivo como suaves, ondulados, delgados, dóciles y fáciles de ordenar. Antonio, administrador de una barbería, señala que «el cabello de nuestra raza peruana es diferente al cabello de los europeos, en textura, largo, en forma. El cabello por ejemplo de un latino peruano es cabello lacio trinchudo»,

Es importante notar que para la mayoría de barberos y administradores, tanto el pelo peruano como el pelo europeo, son tipos ideales de cabello y no pertenecen de modo exclusivo a una región o país. Más bien, se encuentra una diversidad de cabellos en proporciones diversas en distintos lugares geográficos. El barbero Bruno explica que «son pocos los que tienen el cabello ondulado [en el Perú] porque la gran mayoría tiene el cabello lacio, o lo tiene ondulado o lo tiene trinchudo. No es que haya un tipo determinado de cabello para cada país o cada continente [...]»; sin embargo, a pesar de que la mayoría no tiene el pelo ondulado en el país, «en Europa es donde se marca más la tendencia [...]». Esto es, se impone una tendencia que no está acorde con el tipo mayoritario de cabello en el Perú. Por otra parte, el hecho de que la barba no sea frecuente entre los hombres peruanos resulta relevante, pues permite a un grupo de entrevistados desarrollar narrativas racializadas y culturales que se sustentan en un componente o registro narrativo genético. Enrique (marketero) propone que «si tienes barba eres un poco menos cholo», esto es, alguien bien considerado en la medida en que ser cholo implica ser asociado con características peyorativas fenotípicas atribuidas a la población mestiza o andina. En la misma línea que Enrique, Ronaldo (agente de viajes) comenta que tener barba le permite ser considerado extranjero (algo positivo desde la idiosincrasia peruana), dado que «los Incas [...] no eran barbones», lo que le permite pasar como un turista foráneo, porque no estaría relacionado con esa estirpe peruana. "Yo voy al Cusco, Arequipa, o me hablan en inglés o me dicen: “¿De qué país eres?”. Y yo les digo "no, soy de acá". "No, pero tienes barba"». Es de notar que al entrevistado lo entusiasma el hecho de tener barba, ya que lo aleja se ser considerado cholo. Este tipo de argumentación muestra claramente un registro narrativo racializado y jerarquizado. Cabe resaltar, sin embargo, que los entrevistados que tienen barba se cuidan de mencionar que no son racistas.

Por otra parte, encontramos un segundo registro narrativo que racializa y jerarquiza el trabajo del cabello y de la barba, al considerar la raza en su acepción cultural y performativa y, por tanto, como una categoría inestable, relativa y

4 Cabellos lacios, erizados y rebeldes. 
temporal. «Puta qué cholo ese corte», expresa visceralmente Enrique (marketero) cuando describe el corte urbano de un jugador de fútbol peruano. Esto es, se trata de una práctica de choleo tal como describen Bruce (2007) y Nugent (2017). Rubén (abogado) enfatiza que, a fin de cuentas, lo importante es saber escoger un corte «adecuado» para no ser tildado de cholo, lo que él asocia con gentes de barrio o pirañas ${ }^{5}$.

Yo me siento y [mi barbero] ya sabe lo que debe de hacer, es un fade pero no low, es un high, pero no tan high, o sea él sabe el punto exacto. Es un mid fade pero tampoco no tan mid, es entre mid y high, porque si lo hago muy alto se ve muy de barrio y soy abogado. Y creo que ese tipo de cortes tan versátiles se les ve bien a otras personas con otro tipo de características faciales, si eres rubio, blancón, todo te va a quedar de puta madre, si eres más peruanizado, si te sabes un degradado súper alto vas a parecer más piraña que una persona estéticamente correcta, ¿entiendes?

El barbero como intermediario cultural es el que propone los estilos de moda y sugiere - como señalamos - el tipo de corte y tratamiento del cabello y la barba, y, luego el cliente elige según sus preferencias. Lo que encontramos, sin embargo, es el temor a que sus cortes puedan ser tildados de cholos, esto es, desde su perspectiva, vulgares, estrafalarios o identificables con personas poco distinguidas y valoradas socialmente. Como señala Rubén, hay personas «estéticamente correctas» y otras «peruanizadas».

Cabe resaltar, entonces, que más allá de las características intrínsecas del cabello y de la barba, el cliente, con la ayuda del barbero, está en la capacidad de evitar ser tildado de cholo. Para ello el cliente debe gestionar correctamente su cabello y eventualmente su barba y alinearlos con su ropa y su cuerpo. Es, a fin de cuentas, estar bien alineado para estar bien presentado, proceso más difícil si se trata de una persona «estéticamente incorrecta».

\section{ANÁLISIS Y REFLEXIONES}

Estar bien presentado - desde la perspectiva de los entrevistados-consiste en alinear las características corporales de la persona, el tipo de ropa que usa y el trabajo de su cabello o la barba. Es, en otras palabras, lograr que en su conjunto muestren orden, aseo y sofisticación. Para un grupo de entrevistados, el proyecto de estar bien presentado podría ser prerrogativa de hombres de cualquier clase

\footnotetext{
$5 \quad$ Ladronzuelos de corta edad.
} 
social. Ello - indican — porque, para ejercitar el cuerpo, se encuentran gimnasios en todos los distritos limeños, se puede conseguir ropa de moda a precios accesibles en mercados y tiendas por departamentos, y, por último, se puede encontrar barberías de barrio cuyos precios son asequibles.

Cabe señalar, entonces, que en términos conceptuales es posible establecer una diferencia entre tener buena presencia y estar bien presentado. En el primer caso se trata de una condición adscrita de la persona con reminiscencias neocoloniales. En el segundo, es el resultado de un proyecto democrático, esto es, de una habilidad que cualquier hombre puede desarrollar relacionada con la responsabilidad y disciplina (Centurión, 2021).

Asimismo, la buena presencia sería inherente a una condición fenotípica blanca y de clase social media alta. Esto es, se tiene buena presencia. Por su parte, estar bien presentado es un trabajo sobre la apariencia que cualquier hombre - al margen de su clase social — puede emprender. No podemos concluir, sin embargo, a partir de las entrevistas, que ambas condiciones se encuentren empíricamente separadas. Tampoco podemos afirmar que los entrevistados aprueban la condición democrática del trabajo de los hombres en el logro de estar bien presentados. Ello, pues, se puede deber a una declaración de principios de corrección política en el contexto de la entrevista que llevamos a cabo. Lo que sí es dable señalar es que asistir a una barbería clásica es una experiencia que marca fronteras de clase debido al costo de sus servicios y a su puesta en escena. En las barberías clásicas se presta atención a los gustos de la clase media alta en lo referente a la decoración, la música, el vestuario de los barberos y los servicios que brindan, e incluso, a los temas y al lenguaje que los barberos deben emplear para conversar con los clientes a quienes identifican como de otra de clase social.

Los hombres que entrevistamos son profesionales con carreras en ascenso, $\mathrm{y}$, como ellos señalan, requieren una buena imagen para dar cierto impulso a sus carreras. No en vano los entrevistados asocian el cuidado de su apariencia con interacciones sociales, laborales y afectivas satisfactorias, exitosas y prometedoras. Para lograr una buena apariencia y estar bien presentados, los entrevistados consideran al cuerpo como un proyecto que se debe intervenir a partir de la dieta, el ejercicio, el vestido y el tratamiento del cabello y la barba.

A los entrevistados les queda claro que su generación es vanidosa si la comparan con la de sus padres y abuelos, y lo valoran en tanto les permite mayor discrecionalidad sobre la manera de tratar su cuerpo para estar en el mundo. La valía de la vanidad tiene una relevancia significativa en la vida de los hombres en la medida en que les permite creatividad y expresión de la individualidad y, 
por tanto, satisfacción, goce, seguridad y autoestima. El capital simbólico que adquieren con el trabajo de la apariencia a partir del cabello y de la barba los lleva a consolidar su capital erótico, tal como describe Hakim (2011). Esto es, la conjunción de la belleza desfeminizada, el sex appeal, habilidades sociales, vivacidad y estilo. En un cierto sentido podríamos señalar que cortar o tratar el cabello o la barba pueden «cambiar la vida», como señalaba un entrevistado.

A pesar de los sentimientos positivos, autoestima y goce que genera el trabajo de la apariencia, los entrevistados se enfrentan con el temor de ser feminizados por el mismo deseo de trabajar la apariencia. Ese temor, sin embargo, es conjurado en las barberías en la medida en que en esos contextos se desfeminiza la belleza, masculinizando así el trabajo de la vanidad. En ese sentido, las barberías proponen una forma de encauzar la vanidad masculina en tanto una manera de constituir cuerpos y corporeidades.

La gestión del cabello y de la barba que realizan los barberos con los clientes de barberías con los que conversamos muestra la apropiación de una estética de la suavidad y la sensibilidad, a contracorriente de la estética de la rudeza de sus padres y abuelos, según mencionan. Para Messner (1993), aquel tipo de trabajo de la apariencia no se correlaciona necesariamente con un cambio de sustancia, esto es, con la disminución del sexismo o con la erosión del patriarcado. Esto es, se trata de dos órdenes de realidad distinta. En línea con dicha perspectiva, Bridges y Pascoe (2014) acuñan el concepto de masculinidades híbridas, es decir, hombres con masculinidades dominantes (blancas, heterosexuales y de clases acomodadas) que incorporan prácticas no hegemónicas (femeninas, gais y racializadas, etc.), sin perder su hegemonía frente a otros hombres y mujeres. Se trata, a fin de cuentas, de masculinidades heteronormativas reelaboradas en las que los hombres se encuentran atentos a su imagen corporal en sociedades orientadas al consumo.

En el caso del estudio que llevamos a cabo encontramos que los entrevistados desfeminizan el arreglo de su apariencia participando del ambiente masculinizado de las barberías, consumiendo la publicidad de los productos cosméticos y de aseo «solo para hombres» y las imágenes y estilos de los hombres exitosos (deportistas, artistas, etc.) cuyos cabellos y barbas son referentes. Esto muestra la complejidad de las reflexiones y emociones que se ponen en juego cuando los hombres se acicalan en una barbería. Por un lado, se encuentra el reclamo por el derecho a la vanidad y el arreglo de la apariencia; por otro, se argumenta que no son sexistas ni homofóbicos, y en tercer lugar, se identifican fronteras simbólicas o prácticas de desfeminización. 
Finalmente, resulta menester señalar que las narrativas racializadas sobre el cabello y la barba se construyen bajo dos registros: el primero hace referencia al pelo chuto imposible de peinar, asociado a una raza autóctona o una raza sin barba, esto, en su acepción genética, adscrita, dicotómica y monolítica (esta narrativa fue propuesta principalmente por barberos y administradores de barberías), y el segundo plantea que la raza se asocia con indicadores culturales relacionados con lo cholo en su acepción peyorativa (desordenado, sucio, extravagante y de mal gusto).

Así, el proyecto de los entrevistados en términos de la construcción de su estilo es evitar tener un corte que parezca cholo. El discurso racializado bajo el segundo registro es un juego de apariencias en el que se intenta tener un buen corte para no parecer cholo, sobre todo «cuando no se es tan blanco». Desde esa misma perspectiva, el corte del cabello y de la barba agregan a la raza inestabilidad y temporalidad; esto es, en la medida en que no se logra mantener un buen corte, se cae en la categoría de cholo. Esto resulta relevante, pues los entrevistados se clasifican en un continuum racial cuyos polos son la raza autóctona peruana y la raza europea blanca. Así, su proyecto es huir de la posibilidad de ser tildados de cholos. A ello se refiere Santos (2014) con «juegos de discriminación», es decir, la manera en que las personas - en contextos particulares - producen narrativas racializadas y la posibilidad de estudiarlas dada la riqueza que transmiten en calidad y profundidad de información. Por otra parte, conjeturamos que la separación de las narrativas racializadas en los dos registros que proponemos en la investigación resulta una estrategia analítica, más que empírica, en la experiencia de vida de los entrevistados. Cabe señalar, sin embargo, que los hallazgos de Fuller (2001) — casi dos décadas atrás - acerca de la asociación de la belleza con el look caucásico (piel clara, ojos azules y cabello rubio) sigue vigente y da cuenta de las diferencias de clase y narrativas racializadas que sostienen las jerarquías sociales en el Perú.

\section{REFERENCIAS}

Alfonso, M., Richter-Appelt, H., Tosti, A., Sánchez Viera, M. y García, M. (2005). The psychosocial impact of hair loss among men: A multinational european study. Current Medical Research and Opinion, 21(11), 1829-1836. https://doi. org/10.1185/030079905X61820

Álvarez Capuñay, S. (2018). Construcción simbólica de la identidad de la mujer peruana a través del cabello [trabajo final de Máster Universitario en Investigación y Creación Artística]. Madrid: Universidad Complutense de Madrid. Facultad de Bellas Artes. 
Barber, K. (2008). The Well-Coiffed Man: Class, Race, and Heterosexual Masculinity in the Hair Salon. Gender \& Society, 22(4), 455-476. https://doi. org/10.1177/0891243208321168

Barber, K. (2016). «Men Wanted»: Heterosexual Aesthetic Labor in the Masculinization of the Hair Salon. Gender \& Society, 30(4), 618-642. https://doi. org/10.1177/0891243216637827

Bridges, T. y Pascoe, C. J. (2014). Hybrid Masculinities: New Directions in the Sociology of Men and Masculinities. Sociology Compass, 8, 246-258. https://doi. org/10.1111/soc4.12134

Bruce, J. (2007). Nos habiamos choleado tanto. Psicoanálisis y racismo. Lima: Universidad de San Martín de Porres.

Cheang, S. y Biddle-Perry, G. (2008). Conclusion. Hair and Human Identity. En G. Biddle-Perry y S. Cheang (eds.), Hair. Styling, Culture and Fashion (pp. 243-253). Londres: Bloomsbury. https://doi.org/10.2752/9781847887191/HAIR0023

Cheong, H. F. y Kaur, S. (2015). Legitimising male grooming through packaging discourse: a linguistic analysis. Social Semiotics, 25(3), 364-385. https://doi. org/10.1080/10350330.2015.10266

Centurión, O. (2021). Barberos en Lima: belleza masculina y la hegemonía visual en torno al pelo y vello facial [Tesis de magíster]. Lima: PUPC. Recuperado de http://hdl.handle.net/20.500.12404/18669

Clarke, L. H. y Griffin, M. (2008). Visible and invisible ageing: Beauty work as a response to ageism. Ageing \& Society, 28, 653-674. https://doi.org/10.1017/ S0144686X07007003

Coad, D. (2008). The metrosexual: Gender, sexuality, and sport. Albany: SUNY Press.

Cox, C. (2008). Foreword. En G. Biddle-Perry y S. Cheang (eds.). Hair. Styling, Culture and Fashion (pp. ix-x). Londres: Bloomsbury. https://doi. org/10.2752/9781847887191/HAIR0001b

Edwards, T. (2003). Sex, Booze and Fags: Masculinity, Style and Men's Magazines. The Sociological Review, 51 (suplemento), 132-146. https://doi.org/10.1111/j.1467954X.2003.tb03607.x

Euromonitor International (n.d.). Retail Value RSP of Men's Grooming in Peru (per capita). Recuperado de https://www.portal.euromonitor.com/

Escalante, J. (1 de noviembre de 2011). El resurgimiento de las barberías en el mundo. El Comercio y ESAN. Recuperado de https://archivo.elcomercio.pe/especial/50ideas-de-negocios/noticias/resurgimiento-barberias-mundo-noticia-1992602

Featherstone, M. (2010). Body, Image and Affect in Consumer Culture. Body \& Society, 16(1), 193-221. https://doi.org/10.1177/1357034X09354357 
Fuller, N. (2017). Peruvian Beauty. Harvard Review of Latin America, XVI, 37-40. Recuperado de http://revista.drclas.harvard.edu/files/revista/files/135653_webcomplete.pdf

Fuller, N. (2001). Masculinidades: cambios y permanencias. Varones de Cuzco, Iquitos y Lima. Lima: Fondo Editorial de la Pontificia Universidad Católica del Perú.

Gill, R., Henwood, K. y McLean, C. (2005). Body Projects and the Regulation of Normative Masculinity. Body \& Society, 11(1), 37-62. https://doi. org/10.1177/1357034X05049849

Glaser, B. y Strauss, A. (1967). The discovery of grounded theory: Strategies for Qualitative Research Chicago: Aldine Press. https://doi.org/10.1097/00006199196807000-00014

González Saavedra, M. L. (2013). Entre la predación y la docilidad: padecimiento shawi en la Alta Amazonía [tesis doctoral de Historia Contemporánea]. Universidad Complutense de Madrid, Facultad de Geografía e Historia.

Grogan, S. (2010). Promoting Positive Body Image in Males and Females: Contemporary Issues and Future Directions. Sex Roles, 63, 757-765. https://doi. org/10.1007/s11199-010-9894-z.

Hakim, C. (2011). Erotic capital: The power of attraction in the boardroom and the bedroom. Nueva York: Basic Books.

Hall, M. (2014). Metrosexual masculinities. Hampshire: Palgrave Macmillan. https:// doi.org/10.1057/9781137404749

Janampa, A. S. (2015). Rubias «al pomo» o el blanqueamiento de la belleza en sectores altos de Lima. En L. Kogan (ed.), Belleza, musculatura y dolor: etnografias de cuerpos en Lima. Lima: Fondo Editorial de la Universidad del Pacífico.

Junyi, W. (2019). Ritual de la Pelazón (Worecüchiga) de los Ticunas en el proceso de Evangelización desde el siglo $X X$ [tesis de magíster en Antropología con mención en Estudios Andinos]. Pontificia Universidad Católica del Perú, Facultad de Ciencias Sociales.

Kogan, L. y Galarza, F. (2014). ¿Discriminas o te discriminan?: un análisis de las percepciones de universitarios de cuatro ciudades del Perú. Lima: Fondo Editorial de la Universidad del Pacífico.

La Cámara (5 de marzo de 2020). COPECOH: sector Cosméticos crecería entre 2,6\% y $3,2 \%$ el 2020. Recuperado de https://lacamara.pe/copecoh-sector-cosmeticos-ehigiene-creceria-entre-31-y-41-el-2020/

McDowell, L. (2005). The men and the boys. Bankers, burger makers and barmen. En B. van Hoven y K. Hoerschelmann (eds.), Spaces of Masculinities (pp. 17-28). Londres: Routledge. 
Messner, M. (1993). «Changing Men» and Feminist Politics in the United States. Theory and Society, 22(5), 723-737. https://doi.org/10.1007/BF00993545

Moore, L. J. y Casper, M. J. (2015). The body: Social and cultural dissections. Nueva York: Routledge. https://doi.org/10.4324/9780203556832

Neila Boyer, I. (2006). «El samay», «el susto» y el concepto de persona en Ayacucho, Perú. En G. Fernández Juaréz (coord.), Salud e interculturalidad en América Latina: antropología de la salud y crítica intercultural (pp. 187-216). Ciudad Real: Universidad de Castilla-La Mancha.

Nugent, G. (2017). El laberinto de la choledad. Lima: Universidad Peruana de Ciencias Aplicadas.

Santos, M. (2014). La discriminación racial, étnica y social en el Perú: balance crítico de la evidencia empírica reciente. Debates en Sociología, 39, 5-37. Recuperado de http://revistas.pucp.edu.pe/indexhp/debatesensociologia/article/view/10966

Santos, N. C. y S. Pereira (2019). Beard, hair, and mustache: Consumption and masculinities in barbershops. Revista de Administração de Empresas, 59(3), 183-194. https://doi.org/10.1590/S0034-759020190304

Simpson, M. (1994). Male Impersonators: Men Performing Masculinity. Londres: Cassell.

Synnott, A. (1987). Shame and Glory: A Sociology of Hair. The British Journal of Sociology, 38(3), 381-413. https://doi.org/10.2307/590695

Ocejo, R. E. (2017). Masters of craft: Old jobs in the new urban economy. Princeton: Princeton University Press. https://doi.org/10.23943/princeton/9780691165493.001.0001

Oldstone-Moore, C. (2017). Of beards and men: The revealing history of facial hair. Chicago: The University of Chicago Press.

Vargas Benavente, R. (2016). Del cholo de mierda al cholo power. Discriminación, prototipos y cambio semántico en el español del Perú [tesis de doctorado en Literatura - Estudios Hispánicos]. Université de Montréal. Faculté des Arts et des Sciences.

Villa, J. (2015). Cuerpo, masculinidad y estilo en jóvenes de sectores altos de Lima. Debates en Sociología, 40, 61-91. Recuperado de http://revistas.pucp.edu.pe/ index.php/debatesensociologia/article/view/13859

Watson, J. (2000). Male bodies: Health, culture and identity. Buckingham: Open University Press.

Weiner, N. (2019). Fashion vs style: The repudiation of fashion in online menswear communities. International Journal of Fashion Studies, 6(1), 3-23. https://doi. org/10.1386/infs.6.1.3_1 